\title{
As Múltiplas Tarefas e Atividades Interferentes em Centrais de Atendimento Telefônico
}

\section{Temporal Management of Multiple Tasks and Intervenient Activities in Call Centres}

Grupo de Pesquisa em Ergonomia para o setor de Petróleo e Gás, LABER/ERGONPROJETOS/INT
Este artigo trata do estudo da gestão temporal das múltiplas tarefas e atividades interferentes em situações reais de trabalho. Através do estudo da intensificação do trabalho em Centrais de Atendimento, constatamos que os operadores, para conseguirem executar suas atividades, sobrepõem fases do atendimento, optando por executar múltiplas tarefas de maneira a tentar comprimir o tempo de atendimento. Outrossim, foi constatado que ocorre a sobreposição de chamadas, gerando atividades interferentes à chamada principal e que devem ser gerenciadas pelos atendentes.

Palavras-chave: gestão temporal, dupla atividade, atividade interferente.

The aim of this article is to understand the forms of temporal management of multiplicity and intervention between tasks. The results obtained in the study of the intensification in call centers show that the operators, in order to be able to do their activities, overlap the phases of the service, doing multiple tasks to compress the time spent on service. On the other hand, it was found that there is an overlapping of calls which leads to intervenient tasks to the main call that must be managed by the operators.

Keywords: temporal management, multiple tasks, intervenient activities. 


\section{Introdução}

Este artigo aborda o trabalho humano nas Centrais de Atendimento Telefônico, interessando-se pela gestão temporal das múltiplas tarefas e atividades interferentes realizadas pelos atendentes, considerando as seguintes definições abaixo:

- Atividades múltiplas, tarefas com ocorrência prevista, possibilidades de antecipação, possibilidade de repartição de tempo entre diferentes tarefas dos operadores;

Atividades interferentes; atividades com ocorrência imprevista, impossibilidade de antecipação, necessidade de diferentes modalidades de gestão e regulação temporal (Gadbois, 1991; Santos, 2002).

O trabalho humano nestes centros, como em outras situações na área de serviços, está sujeito à grande variabilidade, ao dinamismo e à imprevisibilidade, características próprias de sistemas complexos (Pavard, 1999; Hutchins, 1991, apud Nardi, 1996). A intensificação do trabalho exige do operador a execução simultânea de tarefas múltiplas e que concorrem entre si interferindo na atividade principal.

Existem três modelos teóricos que estão diretamente relacionados às situações de tempo compartilhado, nas quais existe a ocorrência imprevista de tarefas sem a possibilidade de antecipação (Ouni, 1998):

- o modelo do canal único que se fundamenta na idéia da transmissão de uma única informação por vez. O conceito foi desenvolvido a partir da teoria das comunicações de Shannon \& Weaver (1949), apud Ouni, enriquecida por Broadbent (1958, apud Ouni, 1998) e depois por Welford (1967, apud Ouni, 1998);

- o modelo de múltiplos processadores desenvolvido por Reynolds \& Coll (1972, apud Ouni, 1998) que propõe a existência de múltiplos processadores de tratamento, independentes e especializados, acoplados a um processador global organizacional que anuncia um modelo de recursos múltiplos;

- a teoria do tratamento controlado e do tratamento automático (Schiffrin \& Schneider, 1977, apud Ouni, 1998) é modelo da memória fundamentado praticamente sobre a metáfora do computador. Esse modelo distingue dois processos de tratamento: um processo automático e um processo controlado. Logo, a interferência do tratamento pode ser provocada quando as exigências de tratamento controlado de tarefas ocasionam a ultrapassagem da capacidade de concentração (Camus, 1988, apud Ouni, 1998).

Essas três abordagens (teoria do canal único, recursos múltiplos e tratamento automático e controlado) consideram o homem como um sistema de tratamento em termos de canal, de recurso de atenção. $A$ interferência do tratamento é explicada pela capacidade do canal e pela sobreposição de recursos. Mas, segundo Montmollin (1984), dado o comportamento mental de um operador, as tomadas de informações não podem estar reduzidas a um modelo puramente quantitativo de um canal mais ou menos saturado. $O$ autor mostra que para sujeitos com competências diferentes existe uma variação na tomada de informações (qualitativa e quantitativamente). Logo, a carga não é a mesma para ambos. Segundo Ouni (1998), as modalidades experimentais descritas anteriormente tomam como base as entradas e as saídas periféricas (modalidades verbais/ espaciais, visão e audição) muito controladas, que permitem confirmar e inferir hipóteses somente sobre a base de raciocínios indiferentes, elaborados a partir do desempenho. A escala de tempo necessária à execução de tarefas observadas nas pesquisas é da ordem de algumas frações de segundo, o que não é pertinente em relação aos objetivos dos estudos da gestão temporal de tarefas interferentes. E, ainda, a avaliação da carga mental pela degradação do desempenho das tarefas principais e secundárias propostas não diz nada sobre as estratégias dos sujeitos que são obrigados a obter um resultado. É necessário, então, abordar a questão da capacidade da regulação da carga de trabalho pelos operadores em situação de dupla atividade.

\section{Metodologia}

Nossa pesquisa partiu da hipótese de que a intensificação das Centrais de Atendimento 
e a redução dos tempos de atendimento provocam a execução de dupla ou tripla atividade (falar, escrever e navegar) pelos atendentes. A importante atividade cognitiva tem como conseqüência a rigidez postural e os riscos de doenças ocupacionais. Para validar essa hipótese, realizamos um estudo de caso sobre venda e uso de telefones celulares para populações de baixa renda no nordeste do Brasil, procedendo a análise ergonômica do trabalho (AET) nas Centrais de Atendimento.

Foram realizadas, em uma etapa inicial, observações de um grupo de operadores dividido em três subgrupos distintos $(1,2,3)$, segundo o seu nível de aprendizagem (vide Tabela 1).

Tabela 1 Primeiro Grupo Observado. Subgrupo I: operadores experientes (1 A e 1B);

Subgrupo Il: operadores menos experientes (2A e 2B);

Subgrupo Ill: operadores principiantes (3A e 3B).

\begin{tabular}{c|c|c}
\hline \multicolumn{3}{c}{ PRIMEIRO GRUPO OBSERVADO } \\
\hline Subgrupo I & Tempo de Serviço & $\begin{array}{c}\text { Tempo de } \\
\text { Observação }\end{array}$ \\
\hline Operador 1A & 3 anos e 6 meses & 1 hora \\
Operador 1B & 2 anos e 6 meses & 1 hora \\
\hline
\end{tabular}

\begin{tabular}{c|c|c}
\hline Subgrupo II & Tempo de Serviço & $\begin{array}{c}\text { Tempo de } \\
\text { Observação }\end{array}$ \\
\hline Operador 2A & 13 meses & 1 hora \\
Operador 2B & 10 meses & 1 hora \\
\hline Subgrupo III & Tempo de Serviço & $\begin{array}{c}\text { Tempo de } \\
\text { Observação }\end{array}$ \\
\hline Operador 3A & 6 meses & $\begin{array}{c}1 \text { hora } \\
1 \text { hora }\end{array}$ \\
\hline Operador 3B & 2 meses & 1 . \\
\hline
\end{tabular}

Os atendentes foram selecionados entre aqueles que tinham uma ótima avaliação do trabalho, eram considerados pela empresa como bons funcionários e que concordaram em colaborar com o trabalho de pesquisa. Foram feitos registros sistemáticos do trabatho dos seis atendentes durante uma hora com cada um, no horário de pico (entre 14h e 16h).

Além da observação sistemática do pesquisador que acompanhou toda a navegação das telas, os atendentes foram filmados assim como foi realizada a gravação de todo o conteúdo das comunicações no trabalho. Foram registrados sessenta e seis atendimentos ao longo de seis horas de observações.

\section{Resultados}

A atividade do operador da $\mathrm{CA}$, devido à concepção da tecnologia e ao processo de trabalho, envolve:

- 0 atendimento a chamadas em continuidade e a resolução de problemas;

- a alimentação do banco de dados do cliente, inserindo o histórico da chamada (memo);

- a atualização pessoal em relação às mudanças e aos novos processos.

\section{- Atendimento Construído por Fases}

A partir dos dados levantados em nosso estudo de caso (vide Tabela 2), um atendimento é composto por fases. São elas:

Tabela 2 Fases Prescritas do Atendimento.

\begin{tabular}{|c|c|}
\hline Fase 2 & $\begin{array}{l}\text { Colocação / demanda / checagem das } \\
\text { senhas }\end{array}$ \\
\hline Fase 3 & Procura no sistema \\
\hline Fase 4 & Resolução e negociação do problema \\
\hline Fase 5 & $\begin{array}{l}\text { Formatação da ação / registro do } \\
\text { memo }\end{array}$ \\
\hline Fase 6 & Fechamento da chamada \\
\hline
\end{tabular}

\section{A Regulação da Execução das Fases}

De acordo com o problema a resolver, dos fatores advindos do contexto e do grau de pressão do tempo médio de atendimento, os operadores elaboram estratégias de ação, antecipam ou não algumas fases de atendimento ou prolongam outras. $O$ registro do histórico da chamada pode também ser antecipado ou não e preferencialmente ser realizado durante a chamada, de modo a não se superpor à próxima chamada, que terá conteúdo diferente. Os atendentes gerenciam a ordenação e a execução das fases de atendimento, sendo que o contexto tem uma forte influência sobre a determinação das estratégias adotadas. A partir do modelo a seguir (Figura 1) podemos observar a dinâmica descrita através das principais ações que os operadores (navegação, fala com o cliente, registro do histórico da chamada) devem executar para cumprir as fases do atendimento. 
Como mencionamos, cabe ao operador gerenciar a execução das fases em um ambiente de forte pressão temporal. O operador adotará várias estratégias para solucionar os problemas.

\section{A Gestão Temporal das Ações}

A gestão das principais ações realizadas durante $o$ atendimento (escrever, falar e navegar) é feita segundo as categorias a seguir descritas, que são adotadas ao longo dos atendimentos. A análise quantitativa das seqüências de ações principais (navegar, falar, escrever) mostrou as quatro modalidades básicas de gestão que existem e como essas são variáveis, a saber:

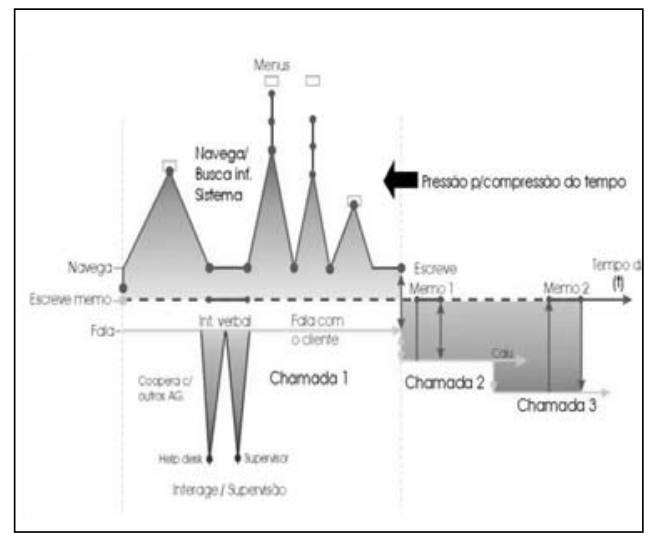

Figura 1 Representação da dinâmica da execução de três ações principais ao longo do atendimento (fala, escreve e navega) e da sobreposição das chamadas.

1) Gestão seqüencial das ações (navegar, escrever, falar com o atendente):

Nesta, o operador executa ações seqüencialmente e interrompe a fala do usuário para não ter que executar dupla atividade (fala $\mathrm{e}$ navega, fala e escreve).

O atendente pode ter dois tipos de gestão do registro do memo:

- execução posterior do histórico (memo): - operador deixa o memo para ser executado no final da ligação, dando prioridade à navegação e a do levantamento de um quadro da realidade do cliente (perfil do cliente);

- execução antecipada do histórico (memo): o atendente antecipa a execução do memo e pode iniciá-lo no mo- mento em que interrompe a navegação ou nos tempos de espera, podendo fragmentá-lo.

2) Gestão simultânea das ações dentro da mesma chamada (dupla tarefa com o mesmo conteúdo).

Neste caso, o operador executa dupla tarefa para acelerar o atendimento:

- navega ao mesmo tempo em que fala com o cliente (Fase 2), seja na fase de colocação da demanda, seja na fase de resolução do problema (fase 4);

- escreve ao mesmo tempo em que fala com o cliente, seja para execução do memo, seja para fazer anotações.

Também nesse caso ele pode ter dois tipos de gestão do registro do memo:

- execução posterior do histórico (memo): o operador deixa o memo para ser executado no final da ligação, dando prioridade à navegação e ao levantamento de um quadro da realidade do operador;

- execução antecipada do histórico (memo): o atendente antecipa a execução do memo nos momentos em que interrompe a navegação ou nos tempos de espera, podendo fragmentá-lo.

3) Gestão simultânea das ações com conteúdos diferentes (executa dupla tarefa com conteúdos diferentes).

O operador executa dupla atividade (ao mesmo tempo em que fala com o cliente, ele navega), pede ao cliente para repetir informações que já estão na tela para ganhar tempo enquanto registra o memo, ou interrompe o cliente e passa por uma gestão seqüencial, ou passa para a gestão futura de recuperação do memo.

4) Gestão de recuperação futura do memo:

- $\circ$ atendente não conseguiu resolver o problema, faz anotações, vai tentar recuperá-lo quando tiver tempo;

- deixa o memo para ser feito nos períodos com menos demanda de atividade, quando esse exige uma justificativa ou um detalhamento maior. 
Ou seja, nas situações 1, 2 e 3 analisadas, ele pode realizar a gestão seqüencial, a gestão simultânea ou a gestão de recuperação futura do memo. A adoção dessas gestões é comandada pela imposição temporal.

A gestão seqüencial é utilizada quando a fila é menor; quando se cria um impasse no decorrer do atendimento, como, por exemplo, quando o usuário não entende algo e ele deve parar para repetir a explicação; na negociação de alternativas de pagamento; quando o cliente está estressado; quando ele não depende de informações da tela e não deve escrever o memo.

A gestão simultânea com um mesmo conteúdo é utilizada para acelerar o atendimento ou quando o atendente quer antecipar a formulação da demanda e a compreensão do problema do cliente.

A gestão simultânea com conteúdos diferentes é utilizada quando $o$ atendente deve recuperar o atraso, a sobreposição de uma chamada a outra, considerando ainda a sua vontade de acelerar ou não a chamada.

A gestão de recuperação é utilizada quando ele não consegue registrar o memo porque não houve tempo ou porque o memo irá demandar uma maior elaboração devido a problemas mais complexos. Ou seja, o ritmo de trabalho é variável, podendo o operador acelerar ou não o processo de atendimento em determinados períodos e utilizar uma ou outra gestão temporal.

Apresentaremos aqui os dados levantados em campo que nos permitirão compreender o uso de diferentes modos de gestão temporal pelo operador. Iremos considerar os seguintes índices:

\section{a) Freqüência de chamadas com dupla tarefa.}

A partir dos dados obtidos, verificamos inicialmente que em grande parte das chamadas todos os operadores executam dupla tarefa (falam e navegam simultaneamente, ou falam e escrevem ao mesmo tempo) dentro de um processo de aceleração do trabalho. Ou seja, em mais de $57 \%$ das chamadas, para todos os operadores, constatamos a dupla atividade.

\section{b) Percentual do tempo de trabalho em dupla tarefa.}

Em relação ao percentual do tempo de trabalho em que os operadores executam dupla tarefa, temos os quantitativos referentes ao:

- percentual de execução de dupla atividade (fala e navega) em relação ao tempo total do atendimento e ao tempo total de navegação;

- percentual de dupla atividade (fala e escreve) em relação ao tempo total de atendimento e ao tempo total de registro do memo.

Verifica-se pelos resultados encontrados que a dupla atividade é uma importante estratégia utilizada para a execução da atividade para fazer face às prescrições feitas pela empresa, permitindo encurtar os tempos de atendimento.

A partir da quantificação do percentual de dupla atividade para os três subgrupos (1A e $1 B, 2 A$ e $2 B, 3 A$ e $3 B$ ), verificamos a importância do processo de busca de informação ou navegação no sistema em relação ao tempo total de atendimento, que varia de $41,4 \%$ a $73,0 \%$ do tempo total de atendimento. Esse dado reflete também o grau de imobilização do corpo $156,3 \%$ a $83,9 \%$ dos operadores têm ação direta sobre o sistema informatizado). A presença de dupla atividade (fala e navega) equivale de $27,0 \%$ a $49,8 \%$ do tempo total da ligação.

O tempo de digitação ("com cola ou sem cola do memo") é de $6,4 \%$ a $24,0 \%$ do tempo total das chamadas e a presença de dupla atividade (fala e escreve) varia de 2,3\% a $10,0 \%$ do tempo total.

Para uma melhor compreensão, apresentamos o resumo dos dados na Tabela 3, que nos leva a concluir que:

- todos os operadores executam dupla atividade. Os mais experientes falam e navegam ao mesmo tempo em um percentual de tempo maior (33,1\% a $49,8 \%$ ).

- a atividade de fala e a digitação são verificadas para todos os atendentes, nos três grupos, entretanto nem todos colam memos padrões. Em 0\% a 57,0\% das chamadas existe colagem do memo. 
Tabela 3 Percentual do tempo total de trabalho no qual os atendentes (1A e 1B, 2A e $2 B, 3 A$ e $3 B$ ) realizam dupla atividade (fala e navega) em relação ao tempo total de navegação. Percentual do tempo total de trabalho em que os atendentes (1A e 1B, 2A e 2B, 3A e 3B) executam dupla atividade (fala e escreve) em relação ao percentual de tempo total de execução do memo.

\begin{tabular}{|c|c|c|c|c|c|c|}
\hline \multirow{2}{*}{ Atividades } & \multicolumn{2}{|c|}{ Subgrupo 1} & \multicolumn{2}{|c|}{ Subgrupo 2} & \multicolumn{2}{|c|}{ Subgrupo 3} \\
\hline & Atendente $1 \mathrm{~A}$ & Atendente 1B & Atendente $2 \mathrm{~A}$ & Atendente 2B & Atendente $3 \mathrm{~A}$ & Atendente 3B \\
\hline Fala e Navega & $49,8 \%$ & $33,1 \%$ & $32,9 \%$ & $30,5 \%$ & $30,6 \%$ & $27,0 \%$ \\
\hline $\begin{array}{l}\text { (A) Tempo total de } \\
\text { navegação }\end{array}$ & $69,5 \%$ & $41,4 \%$ & $49,9 \%$ & $73,0 \%$ & $51,0 \%$ & $65,0 \%$ \\
\hline Fala e escreve & $2,3 \%$ & $10,0 \%$ & $5,3 \%$ & $6,5 \%$ & $4,0 \%$ & $3,5 \%$ \\
\hline $\begin{array}{l}\text { (B) Tempo total de } \\
\text { registro do memo }\end{array}$ & $14,0 \%$ & $24,0 \%$ & $6,4 \%$ & $8,5 \%$ & $10,5 \%$ & $9,9 \%$ \\
\hline $\begin{array}{l}\text { Somatório do tempo } \\
\text { (A) + (B) }\end{array}$ & $83,9 \%$ & $65,4 \%$ & $56,3 \%$ & $81,5 \%$ & $61,5 \%$ & $74,9 \%$ \\
\hline
\end{tabular}

c) Freqüência de interferência das chamadas e freqüência de execução de dupla atividade nas situações de interferência em relação ao número total de atendimentos

A partir da observação do trabalho, verificamos também o percentual de sobreposição de chamadas no número total de atendimentos. No subgrupo 1 (1A e 1B), de operadores experientes, constatamos uma maior freqüência de chamadas sobrepostas.

Na Tabela 4 podemos também observar, segundo diferentes operadores, o percentual do número total de atendimentos em que se executa a dupla tarefa (escrita e fala) em re- lação ao percentual do número de atendimentos nos quais existem chamadas sobrepostas.

Podemos verificar que os operadores experientes têm o maior número de sobreposição de chamadas, o que reflete seu poder de tratamento destas, conseguindo acelerar seu trabalho e estar exposto a um número maior de interferências. Verificamos que todos os operadores, pelos dados anteriormente relatados, executam dupla atividade durante as fases de interferência, mas no subgrupo 1, de operadores mais experientes, executou-se dupla atividade em $40,0 \%$ a $42,0 \%$ das chamadas com conteúdo diferente.

Tabela 4 Percentual de sobreposições de chamada e percentual de execução de dupla atividade durante a sobreposição de chamadas.

\begin{tabular}{l|c|c|c}
\hline Subgrupos & Atendentes & $\begin{array}{c}\text { \% de chamadas } \\
\text { sobrepostas }\end{array}$ & $\begin{array}{c}\text { \% de chamadas } \\
\text { com execução de } \\
\text { dupla tarefa com } \\
\text { conteúdos diferentes }\end{array}$ \\
\hline Subgrupo 1 & $1 \mathrm{~A}$ & $57,1 \%$ & $42,0 \%$ \\
\hline Subgrupo 2 & 1B & $40,0 \%$ & $40,0 \%$ \\
\hline Subgrupo 3 & 2B & $15,3 \%$ & $15,3 \%$ \\
\hline
\end{tabular}

\section{d) Mapeamento da execução do memo}

Para avaliarmos o mapeamento da execução do memo, tomamos como referência dois parâmetros: quantificamos o início e o fim da digitação do memo em relação ao tempo total da chamada. Para isso, consideramos quatro fases correspondentes ao percentual do tempo total da chamada, assim é possível saber se o memo foi antecipado (considerando seu início em até $1 / 4$ do tempo da chamada), ou se foi realizado ao fim da chamada (a partir de $3 / 4$ do tempo da chamada) durante a mesma ligação com o mesmo conteúdo (antecipação ou gestão posterior), ou extravasado para a próxima ligação com conteúdos diferentes. 
De maneira a facilitar nossa compreensão, os dados foram resumidos na Tabela 5 .

De acordo com os dados anteriores, verificamos que a maior parte dos memos é executada em gestão posterior (de $30 \%$ a $66,7 \%$ ) dentro da própria chamada. O nível de antecipação da execução do memo é menor e não está relacionado com o grau de experiência (de $0 \%$ a $26,7 \%$ ). A não antecipação do memo decorre da necessidade do atendente em navegar em uma fase preliminar da chamada para levantar o perfil do cliente (investigar últimos pagamentos, últimos memos). Quando o conteúdo do atendimento não exige a formação desse qua- dro, ou seja, o problema é de fácil resolução, ele pode antecipar o memo. Logo, sua antecipação pode estar relacionada ao grau de complexidade da resolução do problema.

A partir desses dados verificamos que os operadores mais experientes são aqueles que conseguem admitir um percentual maior de registro de memos a serem feitos em outras ligações, quando esses são executados em dupla tarefa com conteúdos diferentes $(33 \%$ a $57,1 \%$ ) sendo finalizados na chamada consecutiva, ou quando são iniciados na próxima chamada (de $13 \%$ a $14 \%$ para o subgrupo 11.

Tabela 5 Percentual do número total das ligações nas quais os memos executados variam segundo a gestão por antecipação, realizados até $1 / 4$ do tempo da chamada, ou a gestão posterior do memo (a partir de $3 / 4$ do tempo da chamada), e percentual do número total de ligações que são finalizadas na próxima chamada ou quando essas são iniciadas a partir da próxima chamada.

\begin{tabular}{|c|c|c|c|c|c|}
\hline \multirow[t]{2}{*}{ SUBGRUPO } & \multirow[t]{2}{*}{ ATENDENTE } & \multicolumn{2}{|c|}{$\begin{array}{c}\text { CHAMADA } 1 \\
\text { Gestão com o mesmo conteúdo }\end{array}$} & \multicolumn{2}{|c|}{$\begin{array}{c}\text { CHAMADA 2 } \\
\text { Gestão com conteúdos } \\
\text { diferentes/sobreposição de chamadas }\end{array}$} \\
\hline & & $\begin{array}{c}\text { Antecipação } \\
\text { Até } 1 / 4 \text { do tempo } \\
\text { da chamada }\end{array}$ & $\begin{array}{l}\text { Início em gestão posterior } \\
\text { a partir de } 3 / 4 \text { do tempo da } \\
\text { chamada }\end{array}$ & $\begin{array}{l}\text { Finalização em } \\
\text { outra ligação }\end{array}$ & $\begin{array}{l}\text { Início em outra } \\
\text { ligação }\end{array}$ \\
\hline \multirow{2}{*}{1} & $1 \mathrm{~A}$ & $0,0 \%$ & $57,1 \%$ & $57,1 \%$ & $14,0 \%$ \\
\hline & $1 \mathrm{~B}$ & $26,7 \%$ & $40,0 \%$ & $33,0 \%$ & $13,0 \%$ \\
\hline \multirow{2}{*}{2} & $2 A$ & $8,3 \%$ & $66,7 \%$ & $15,3 \%$ & $0,0 \%$ \\
\hline & $2 \mathrm{~B}$ & $20,0 \%$ & $40,0 \%$ & $20,0 \%$ & $0,0 \%$ \\
\hline \multirow{2}{*}{3} & $3 A$ & $18,2 \%$ & $45,4 \%$ & $14,0 \%$ & $7,1 \%$ \\
\hline & $3 B$ & $0,0 \%$ & $30,0 \%$ & $10,0 \%$ & $10,0 \%$ \\
\hline
\end{tabular}

Em relação à gestão futura, foi observado apenas para os operadores $2 \mathrm{~A}$ e $2 \mathrm{~B}$ um memo pendente decorrente de atendimentos com reclamação dos usuários.

\section{e) Colagem do memo e processo de atualização durante $o$ atendimento}

Além de todo o atendimento, o operador deve se atualizar através das caixas de mensagem do Outlook. O índice de consulta ao Outlook varia com o conteúdo da ligação. $\mathrm{Em} 0 \%$ a $33,2 \%$ do total dos atendimentos existe essa consulta.

O atendente pode ou não colar textos existentes ou elaborar seus próprios memos. Os dados a seguir (Tabela 6) mostram que em $33 \%$ a $50 \%$ das ligações os atendentes colam memos existentes. O colar significa também ajustar, adaptar o que foi inserido ao texto que já está escrito. Trata-se na maioria das vezes de enxertar um texto complementar, pois os memos padrões não se adaptam a todas as situações. Foi verificado que apenas um dos atendentes não utiliza essa estratégia e prefere digitar seu próprio texto.

Tabela 6 Freqüência de atendimentos com colagem do memo e com a atualização para operadores diversos.

\begin{tabular}{c|c|c}
\hline FREQÜÊNCIA & COLA & ATUALIZA \\
\hline $1 A$ & $33,0 \%$ & $33,2 \%$ \\
\hline $1 B$ & $57,0 \%$ & $14,2 \%$ \\
\hline $2 A$ & $0,0 \%$ & $21,4 \%$ \\
\hline $2 B$ & $46,0 \%$ & $7,6 \%$ \\
\hline $3 A$ & $35,0 \%$ & $0,0 \%$ \\
\hline $3 B$ & $50,0 \%$ & $20,0 \%$ \\
\hline
\end{tabular}


Não foi possível quantificar os erros durante o registro do memo dada a velocidade da digitação, o que poderá ser fonte de investigação futura, assim como os erros na fala dos operadores. Entretanto, podemos sinalizar a sua existência.

Observamos que no processo de dupla atividade em situação de interferência existem erros na fala, atropelos da fala, troca de sílabas, assim como esquecimento do nome do usuário com o qual se está falando. Existem também erros de digitação, típicos da aceleração da digitação (supressão de letras, digitação da tecla vizinha, duplicação de letras e outros), já mencionados por Santos (1994 e 1996). Esses erros são recuperados durante a execução da dupla tarefa (escreve e fala).

\section{Conclusão}

A partir dos dados levantados, verificamos que em uma atividade com tempo compartilhado o operador é levado a reduzir o tempo de execução de tarefas através da sobreposição dessas, dentro de um contexto no qual existem interrupções e incerteza quanto ao seu surgimento. As tarefas, segundo as exigências de complexidade $e$ as imposições temporais, requerem um funcionamento cognitivo do tipo concreto, operatório e formal. Os novatos não têm as mesmas estratégias de gestão de situações similares que os experientes e não podem se adaptar às mesmas perturbações de maneira idêntica. As variabilidades do desempenho e de qualidade do trabalho na atividade dos operadores constituem igualmente manifestações de diferentes capacidades de regulação. Entretanto, no trabalho de CAs com fortes ritmos, existe uma seleção natural dos atendentes ou a seleção pela própria empresa (os que não suportam o trabalho são demitidos ou pedem demissão). Os dados mostraram que uma aceleração é perseguida pelos atendentes que executam dupla tarefa: navegação simultânea à fala de $27,0 \%$ a $49,8 \%$ do tempo de trabalho, fala simultânea ao registro do memo (escrita) de $2,3 \%$ a $10,0 \%$ do tempo de trabalho. Esses dados são confirmados por outros estudos realizados nas centrais jornalísticas quando foram detectadas também a sobreposição de chamadas e a execução de dupla atividade no atendimento (Santos 1997a).

Pelos resultados obtidos em nosso estudo, o grupo mais experiente consegue admitir um número maior de sobreposição de chamadas (de $40,0 \%$ a $57,14 \%$ das ligações), ou seja, conviver com situações com interferência. Esse mesmo grupo registra mais vezes o memo nas chamadas subseqüentes (em $33,0 \%$ a $57,1 \%$ das chamadas) e em 40,0 a $42,0 \%$ das chamadas executa dupla tarefa com conteúdos diferentes. Nossos dados corroboram com os dados de Ouni (1998) a partir dos estudos sobre a experiência face à gestão de atividades interferentes. Segundo o autor, os operadores experientes suportam mais a interferência. O autor fala também dos estudos experimentais de Shiffrin \& Schneider (1977, apud Ouni, 1998), os quais mostram que o prolongamento da confrontação do sujeito com uma dada situação favorece a passagem do tratamento controlado a um tratamento mais automático, o que torna o uso do processo de gestão mais rápido e mais eficiente. O autor enfatiza também o fenômeno da competência (Leplat, 1977 apud Ouni 1998).

Os resultados obtidos mostram claramente que estamos diante de uma nova taylorização do terciário, que, neste caso, prega o forte controle do tempo médio de atendimento, impondo agora aos trabalhadores grandes exigências mentais e psíquicas.

\section{Referências Bibliográficas}

GADBOIS, C. Les tâches interferentes, un problème caracteristique du travail hospitalier. Soins, no 26 (12), pp. 15-18, 1981.

HUTCHINS, E. Distributed Cognit Airline Cockpit. In: Engestrom \& D. Middleton, eds.
Cognition and communication at work, Cambridge University Press, 1991.

MONTMOLLIN (de), M. L'intelligence de la tâche: élements de l'ergonomie cognitive, Peter Lang, Berne, 189 p. 1984. 
NARDI, B. Activity Theory and HumanComputer Interaction. In: Nardi, B., ed. Context and Conunciousness. Massachusetts: The MIT Press, 1996.

OUNI, R. Modalités de gestion temporelle des tâches interférentes dans le controle de processus indépendants. 1998. Thése de Doctorat. Ecole Pratique des Hautes Etudes, Paris.

PAVARD, B. A Cognitive Engineering Methodology. Working document. GRIC-IRIT. Toulouse: Univ. P. Sabatier, 1999.

SANTOS, V. A. A intensificação do trabaIho nas Centrais de Atendimento. As múltiplas tarefas e atividades interferentes. 2002. Tese de Doutorado em Engenharia da Produção, COPPE/ Universidade Federal do Rio de Janeiro.
O estudo dos centros de controle de atendimento de seguradoras de saúde. Relatório Técnico da ERGON PROJETOS. Rio de Janeiro, 1995.

O estudo da central de atendimento em empresas jornalísticas. Relatório Técnico da ERGON PROJETOS. Rio de Janeiro, 1997a.

A Ergonomia e o atendimento ao público: Resultados obtidos nas Centrais de Atendimento. $4^{\circ}$ Congresso Latino Americano e $8^{\circ}$ Congresso Brasileiro de Ergonomia, Florianópolis, 1997b.

Concepção ergonômica de Salas de Controle. In: Duarte, F. eds. Ergonomia e Projeto. COPPE/RJ, Rio de Janeiro: Lucerna, 2000. 